\title{
A Novel Methodology for Fault Identification of Multi-stage Manufacturing Process Using Product Quality Measurement
}

\author{
Xiaorui Tong ${ }^{1}$, Hossein D. Ardakani ${ }^{1}$, David Siegel ${ }^{1}$, Ellen Gamel ${ }^{1}$ and Jay Lee ${ }^{1}$ \\ ${ }^{1}$ NSF I/UCRC for Intelligent Maintenance Systems (IMS), University of Cincinnati, Cincinnati, OH 45221 USA \\ tongxi@mail.uc.edu \\ davarihn@mail.uc.edu \\ siegeldn@mail.uc.edu \\ gamelee@mail.uc.edu \\ Jay.lee@uc.edu
}

\begin{abstract}
Data-driven modeling and fault detection of multi-stage manufacturing processes remain challenging due to the increasing complexity of the manufacturing process, the lack of structural data, data multi-dimensionality, and the additional difficulty when dealing with large data sets. The implementation of add-on sensors and establishing data acquisition, transfer, storage and analysis has the potential to facilitate advanced data modeling techniques. However, besides the associated costs, dealing with high-volume multi-dimensional data sets can be a major challenge. This paper presents a novel methodology for early fault identification of multi-stage manufacturing processes using a statistical approach. The major advantage of the proposed methodology is its reliance on only the product quality measurements and basic product manufacturing records, given the presence of peer sets. This leads to a feasible fault identification solution in a sensor-less environment without investing costly data collection systems. The developed methodology transforms the end-of-process quality measurements to a process performance metric based on a density-based statistical approach and a peer-to-peer comparison of the machines at one stage of the process. This approach allows one to be more proactive and identify the problematic machines that could be affecting product quality. A case study in an actual multi-stage manufacturing process is used to demonstrate the effectiveness of the developed methodology.
\end{abstract}

\section{INTRODCUTION}

With the increasing productivity of manufacturing systems, multi-stage manufacturing processes are a commonly used

Xiaorui Tong et al. This is an open-access article distributed under the terms of the Creative Commons Attribution 3.0 United States License, which permits unrestricted use, distribution, and reproduction in any medium, provided the original author and source are credited. approach. High throughput and strict product quality specifications require that equipment at each stage work properly to deliver qualified products. Any type of failure that happens during the manufacturing process could result in process inefficiencies, productivity reductions, and lower product quality. Examples of such processes include but are not limited to: (i) the automotive body assembly process that assembles multiple parts at multiple stations; (ii) the machining processes that manufacture parts through multiple operations performed in a series of stages; (iii) the process of drug manufacturing that is composed of a series of unit operations, such as milling, granulation, coating, tablet pressing and others. Modeling and fault detection of these processes normally requires extensive efforts on collecting, handling and analyzing processes and product measurements, which often introduces higher investment in the necessary infrastructure in managing the high-volume multi-dimensional data. Previous research has mostly been focused on developing multi-stage process monitoring methods which necessitate the measurement of product quality at different stages of the process (Asadzadeh \& Aghaie, 2012; Shu \& Tsung, 2000; Tsung, Li, \& Jin, 2006; Wolbrecht, D'Ambrosio, Paasch, \& Kirby, 2000; Zhou, Ding, Chen, \& Shi, 2003; Zhou, Huang, \& Shi, 2003).

Statistical Process Control (SPC) has been widely employed in industrial operations to detect the changes in the process through monitoring process/quality variables over time and so to determine if the process is in control. SPC charts were first developed to monitor the key product variables in a univariate way (Scouse, 1985). The most commonly used traditional SPC charts include Shewhart, Cumulative Sum (CUSUM), and Exponentially Weighted Moving Average (EWMA) control charts (Rato \& Reis, 2011). Shewhart control chart was first introduced in 1924, and has extensively been used to display the process data and determine whether the process is within the control limits or not. Process data samples from either fixed or variable time 
intervals are taken. Then some statistics are computed and plotted from the samples in each interval (Reynolds, Amin, Arnold, \& Nachlas, 1988). The CUSUM control chart was first introduced by Page (Page, 1954) and has so far been widely used for monitoring the quality of the manufacturing process. In a CUSUM control chart, samples at a fixed or variable time intervals are taken and the cumulative sum of the differences between the mean of the samples and the target mean is calculated and plotted (Reynolds, Amin, \& Arnold, 1990). Unlike the Shewhart and CUSUM control charts which use the current samples for statistical testing, the EWMA chart uses previous values multiplied by a weighting factor. It adds the summation of past measurements to the current measurement (Neubauer, 1997). EWMA has shown to be useful in detecting small shifts in the process mean (Lucas \& Saccucci, 1990).

In processes where multiple quality characteristics need to be monitored, the traditional SPC charts which individually monitor the key quality characteristics are not sufficient (Macgregor \& Kourti, 1995). That led to the development of multivariate control charts such as Hotelling's T2, MEWMA, MCUSUM and PCA (Rato \& Reis, 2011). Hotelling in 1947 developed T2 statistic as a solution for the problem of controlling several variables. The Hotelling control chart is a multivariate extension of the Shewhart chart that takes into account the correlation between the measurements. MEWMA was introduced by Lowry et al. (Lowry, Woodall, Champ, \& Rigdon, 1992) as an extension of EWMA charts. In this approach, the measurement values of EWMA chart are replaced by vectors of measurements. MCUSUM was introduced by Woodall et al. (Woodall \& Ncube, 1985) to replace the implementation of several CUSUM procedures for multivariate processes. Dimensionality reduction using PCA techniques also serves the purpose of considering multiple quality characteristics. PCA is a well-established and commonly used procedure for reducing the dimensionality in the data by projecting it to another space and taking the components which contain maximum variance. By building a PCA model based on historical data while the process is in normal condition, the future behavior of the process can be referenced against the "in-control" process model (Macgregor \& Kourti, 1995).

Once process faults are detected, the immediate challenge becomes how to identify the root cause in order to direct maintenance actions to a specific equipment in a multistage process. Further developments from the traditional SPC methods to address this problem include the regressionadjustment method developed by Hawkins (Hawkins, 1993), and applied in multiple case studies by others. This method regresses quality variables on subsets of the other quality variables and monitor the residuals from the regression models for each stage. An alternative is the cause-selecting method (Asadzadeh \& Aghaie, 2008), where the regression of quality variable is based on the previous stage. Another class of methods for root cause identification steams from the Stream of Variation (SoV). SoV was developed based on previous researches on identifying sources of variation in auto body assembly (Ceglarek, Shi, \& Wu, 1994; Hu \& Wu, 1992; Liu \& Hu, 1997) and was designed to predict and diagnose the dimensional variation in a multi-leveled automotive body assembly system. SoV methodology was further transformed into a State-Space model introduced by Jin et al. (Jin \& Shi, 1999). In this method, —which was originally developed to model error propagation in sheet metal assembly process - the inherent relationships between errors from various sources was developed to describe the variation process throughout the assembly process. Despite the robustness of this approach in the modeling and diagnosis of multistage manufacturing processes, it requires integration of engineering domain knowledge that varies greatly with manufacturing processes of different nature, limiting the application to certain processes such as the auto body assembly, metal machining processes where process faults are fixture error, machining errors etc.

A crucial requirement when applying the above techniques for diagnosing process faults is to have product quality measurements at each stage of the process. The specific quality measurements differ depending on the manufacturing processes, examples include but are not limited to: deviations from the nominal dimensions for machined parts in a machining process, surface quality measurements for machining operations, the concentration of a composite in a tablet during drug manufacturing processes etc. Without tracking the product quality throughout the process at each stage, the SPC and SoVbased methods would not be effective. Despite the rapid advancement in information technology and data processing, the product quality measurement at each stage is not practical in many processes, in extreme cases, only the quality of the finished product is evaluated. Oftentimes, the finished product may have several quality characteristics being measured. It is thus clear that existing methods will not suffice and a multivariate multistage monitoring approach based on only the quality measurements of the finished products needs to be considered.

This paper introduces a novel methodology for fault identification in a multistage environment, given basic product manufacturing records and the presence of peer sets, where only multiple quality characteristics from the final manufacturing stage are available and no expert knowledge is needed. The analysis model for calculating a product inspection metric is developed and utilized for process monitoring. A peer-comparison approach, applicable to a class of manufacturing types, is integrated to isolate process faults to a specific stage. Section 2 introduces the main aspects in implementing this methodology, which include the modeling for the product inspection metric and machine performance metric. Section 3 contains the full details of implementing the developed methodology for an industrial case study and highlights the results from this real-world 


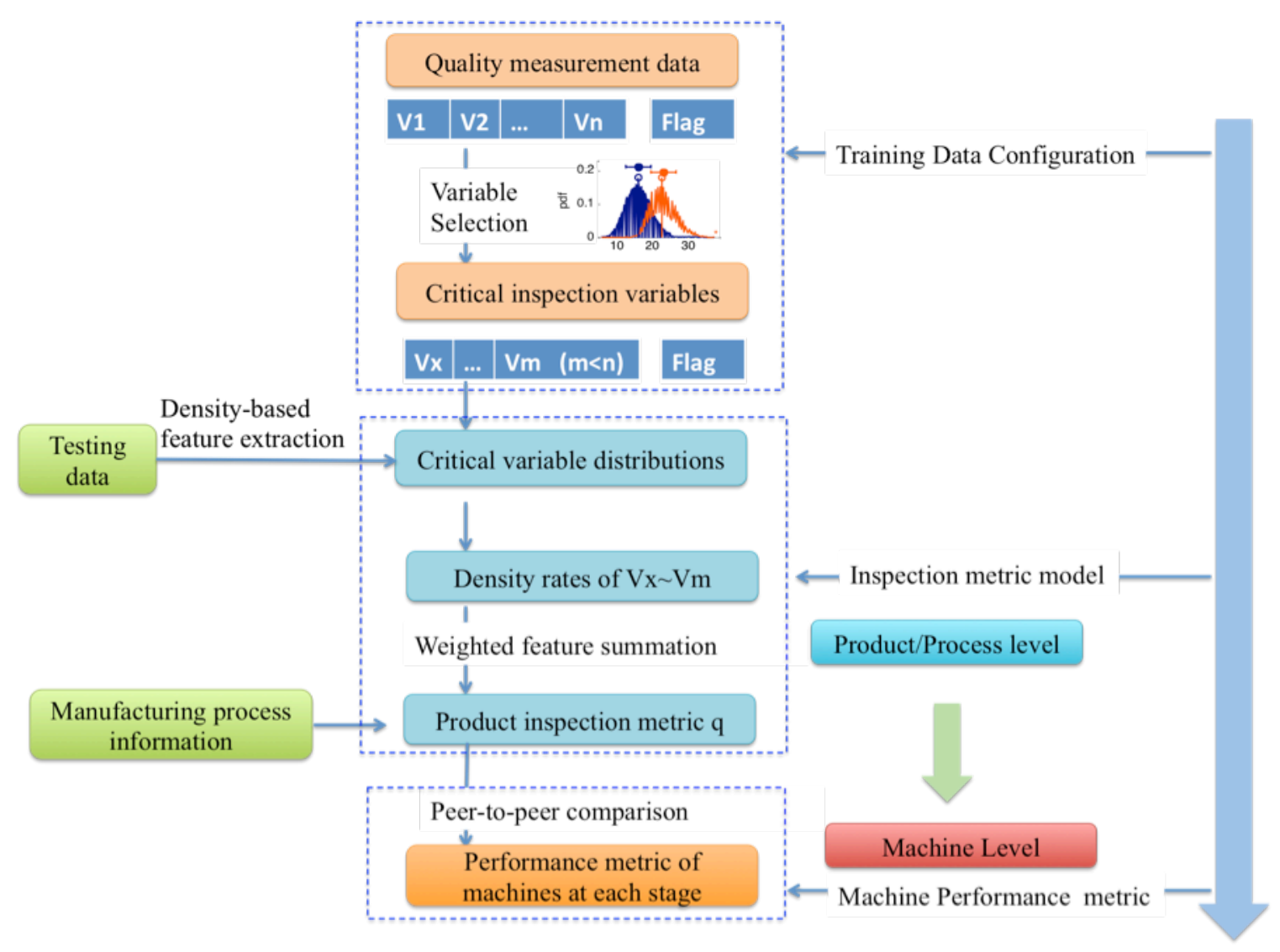

Figure 1. Flowchart of the developed methodology

case study. Conclusions from this study are presented in Section 4, and Section 5 proposes the future work needed for generalizing this methodology.

\section{TECHNICAL APPROACH}

The developed methodology aims to first detect the process anomalies in a multivariate multi-stage manufacturing process and then identify the root causes of the anomalies, using only product quality measurements. To achieve this goal, quality measurement data sets are initially studied to understand the variable correlations and the criticality of each variable. After selecting the critical variables, relationships between the overall product quality level and these variables are modeled to obtain a process performance metric, indicative of the overall multistage process performance. The obtained inspection metrics then are used to detect anomalies of the manufacturing process. Finally, the root causes of detected anomalies are isolated within the machines in the two stages of the process by a peer-to-peer comparison method. The performance metric at machine level indicates the performance of a specific machine at a certain manufacturing stage. A flow chart of the proposed methodology is shown in Figure 1.
While requiring little expert knowledge on the manufacturing processes, the developed methodology can be readily applied to a range of multi-stage processes where product quality measurement contains all continuous variables or discreet variables over a considerable range (so that a distribution can be formed). Manufacturing process information, meaning IDs of machines that have manufactured the product being measured, is also necessary. While the above two requirements can be easily satisfied for many manufacturing process lines, a more stringent premise comes with the peer-to-peer (P2P) comparison, which enables a performance metric at the process level to be converted to a machine level metric. Applying the P2P comparison requires the presence of peer sets in the manufacturing process being studied. The concept of peer sets is introduced in subsection 2.4 where detailed information regarding this important premise can be found.

This section focuses on the key components of this methodology. The steps of the training data configuration are therefore not included in this section. However, these steps are also of great importance since they serve as inputs that are processed downstream by this analysis method. 
Detailed discussion of these steps is presented in Section 3 with the analysis results.

\subsection{Development of Product Inspection Metrics for Anomaly Detection}

When monitoring the process performance through product quality measurements, several issues are identified with the general approach of judging quality solely based on a specification cut-off value (Chan \& Ibrahim, 2004). Some of these concerns include the following:

- Products judged to be of the same quality level could have slightly different quality characteristics as can be seen in Figure 2. Since product quality is indicated with a binary value based on the specification cut-off, the period during which product quality begins to deteriorate while still within the specifications cannot be captured. This period, however, can be used effectively for early detection of performance drift in a manufacturing process.

- For the dataset utilized in this paper, there are multiple sets of quality specification cut-off thresholds depending on the expectations of each customer. This means that if customer $\mathrm{A}$ has higher requirements than customer B, products that are within specifications for customer B can fail the quality test for customer A. Therefore, even the binary values indicating whether a product is within quality specifications or not, may not be completely comparable.

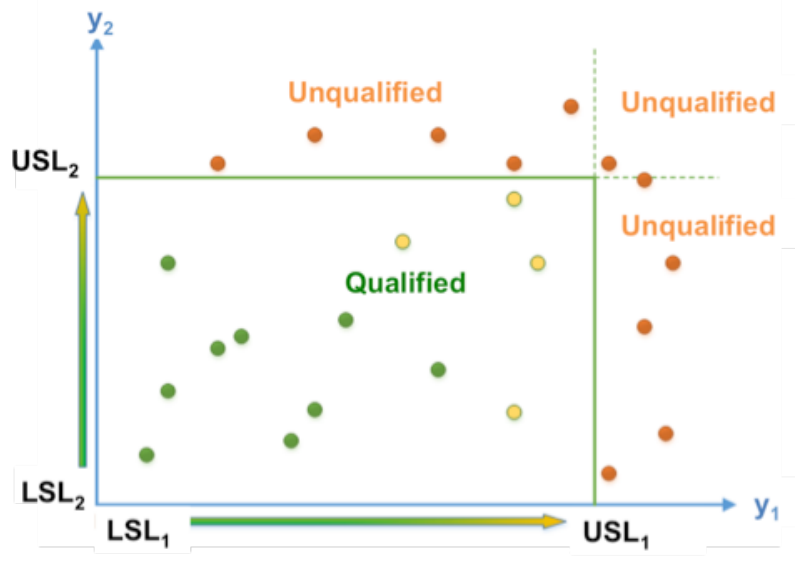

Figure 2. Quality difference between products in the same quality category

Considering such concerns, judging the manufacturing process performance based on product yield may not always be sufficient. Therefore, this paper proposes a novel approach for evaluating overall product quality on a continuous scale that is purely dependent on product inspection measurements instead of product yield. The methodology is based on using a product inspection metric that attributes a score to each product depending on its inspection measurements.

\subsection{Density-based Product Inspection Metric}

In this paper, the concept of density rate is introduced as a feature extraction method for product inspection variables. The extracted density rates for all the critical variables are combined in a weighted summation to obtain the final inspection metric for the manufactured product.

Generally, three types of quality characteristics that have been researched are nominal-the-better (N-Type)(Kapur \& Cho, 1996), larger-the-better (L-Type)(Chan, Ibrahim, \& Lochert, 2005a) and smaller-the-better (S-Type)(Chan, Ibrahim, \& Lochert, 2005b). N-Type means that as the quality measurement gets closer to its targeted mean value, the quality of this product increases. For S-Type, the smaller the measurement is, the higher the quality is. The feature extraction technique proposed in this study is applicable to these three types of quality characteristics in the sense that both N-Type and L-Type can be transformed into S-Type through subtracting from the maximum. To be consistent with the latter case study, quality characteristics of S-Type are considered here. It is worth mentioning that one of the characteristics in the case study belongs to N-Type (Symmetric around 0) and has been transformed to S-Type by taking the absolute value.

For quality measurement of S-Type, smaller value means higher quality. Therefore, features can be extracted by evaluating how small this value is. Figure 3 shows the histogram of the baseline (product with good quality) for one inspection variable and the color lines indicate the positions of this inspection measurement for three product samples. It is apparent that as the sample line moves to the right, its corresponding quality decreases in terms of the one inspection variable.

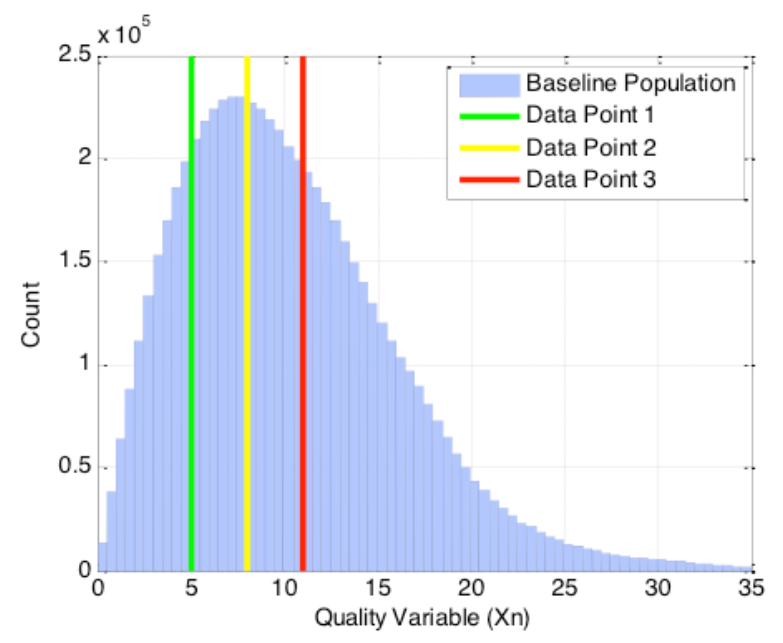

Figure 3. Quality variable locations of three different data points

In terms of distribution, the density rate can be obtained from the cumulative distribution of the baseline data. For a given measurement reading $x_{i}$, its density rate equals one 
minus its corresponding function value on the cumulative distribution curve of the baseline data. Figure 4 shows the empirical Cumulative Distribution Function (CDF) of the distribution shown in Figure 3 along with the density rate values for the three shown samples. The empirical CDF is obtained based on the proportion of population less than the value $\mathrm{x}$ for the corresponding quality variable.

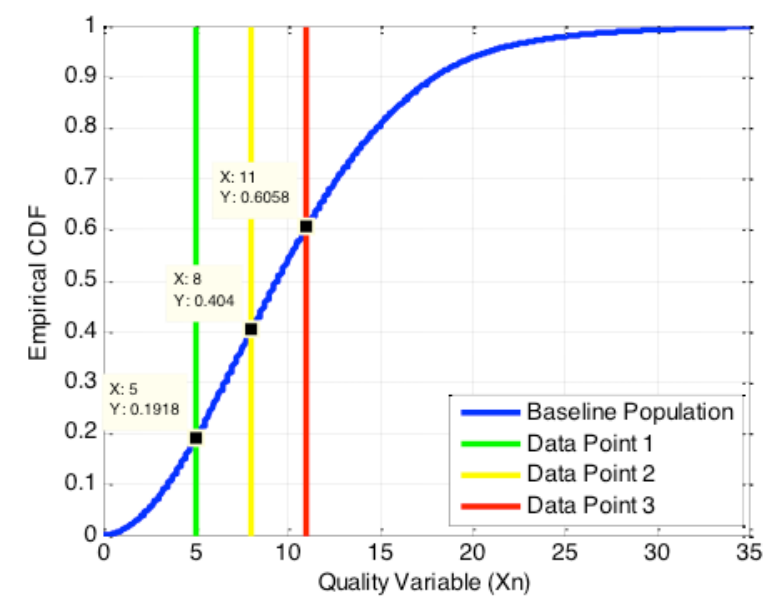

Figure 4. Cumulative function value of the chosen three data points

The relationship between density rate and the cumulative distribution function of the training data distribution is formulated as:

$$
\mathrm{d}_{\mathrm{i}}=1-\mathrm{F}\left(\mathrm{x}_{\mathrm{i}}\right)
$$

where $\mathrm{F}\left(\mathrm{x}_{\mathrm{i}}\right)$ is the corresponding value of the cumulative distribution function for the data point $x_{i}$.

Figure 5 below shows the density rate of two critical inspection variables for product from different categories in the latter case study. Each blue point represents a product that passed the quality check and each red point represents a product that failed the quality check. Although most of the failed products are close to the zero lines, some inconsistency can be observed because of the different quality check thresholds that are set based on different customer expectations. A certain amount of overlap is expected and unavoidable because of the customerdependent quality check standards. However, a key message from Figure 5 is that the unqualified products failed the quality check because their density rate is low in the dimension of $\mathrm{d} 1$ or $\mathrm{d} 2$. There is a tiny portion of unqualified products that have high value for both, therefore, the reason of these products being unqualified is due to low density rates corresponding to other variables. Therefore, this snap shot of the data taught us that a proper combination of the density rates of the quality measurements is an appropriate tool for defining the quality of the products although each quality variable is not adequate individually. As there are multiple quality variables to consider, the next section will be dedicated to fusing these variables to generate a unified metric that represents the quality of the production process.

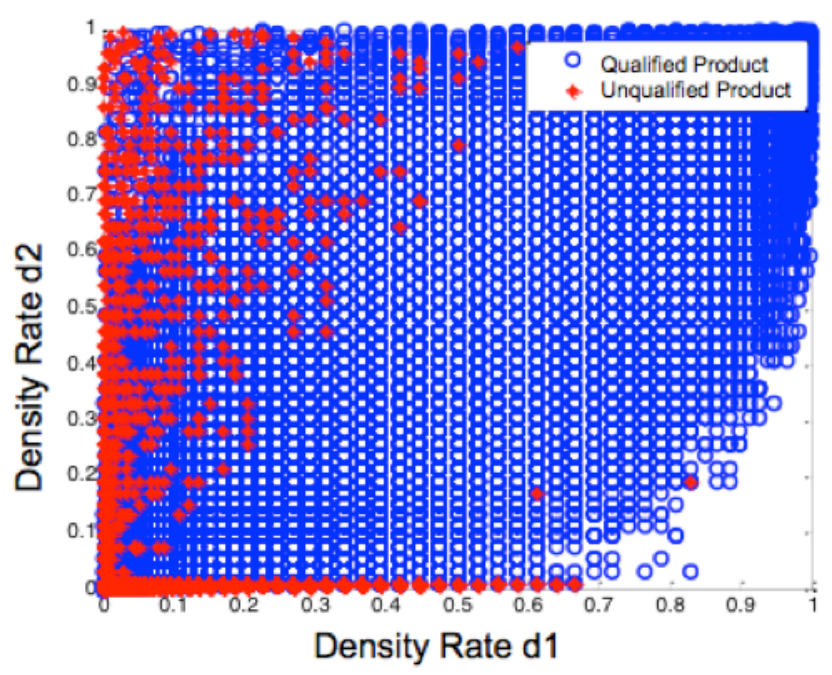

Figure 5. Density rates of two inspection variables

\subsection{Weighted feature summation}

The extracted features from the previous section are combined to build a product inspection metric that rates each product on a continuous scale from 0 to 1 . For defining an appropriate way of building an overall product inspection metric, the following logic is adopted:

- Among all the significant quality variables, the one with the smallest density rate is the main cause of the quality problem for one observation.

- Besides the variable with the smallest density rate, the other variables also contribute to the quality problem with their significances decreasing as the corresponding density rate increases.

Based on the mentioned rules, the product inspection metric should consider the weighted density rates according to their contributions to possible quality problems. Therefore, the overall product inspection metric is designed to be the summation of the density rates for all the significant quality measurements and exponentially weighted according to the value of each density rate. The exponential function $f(x)=x / e^{x}$ is used to weight the density rates, since this function decreases faster approaching 0 and increases slower approaching 1 , which approximates the contributions of the density rates to the overall production inspection metric. Assuming that there are $\mathrm{n}$ significant quality variables for product $\mathrm{a}$, known as $\mathrm{X}_{1}^{\mathrm{a}}, \mathrm{X}_{2}^{\mathrm{a}}, \ldots, \mathrm{X}_{\mathrm{n}}^{\mathrm{a}}$ with density rates of $d_{1}^{a}, d_{2}^{a}, \ldots, d_{n}^{a}$, the density-based quality metric $q_{a}$ can be defined as:

$$
\mathrm{q}_{\mathrm{a}}=\frac{\sum_{\mathrm{i}=1}^{\mathrm{n}} \mathrm{d}_{\mathrm{i}}^{\mathrm{a}} / \mathrm{e}^{\mathrm{d}_{\mathrm{i}}^{\mathrm{a}}}}{\mathrm{n} / \sum_{\mathrm{i}=1}^{\mathrm{n}} \mathrm{e}_{\mathrm{i}}^{\mathrm{d}_{\mathrm{a}}^{\mathrm{a}}}}
$$


The metric defined above thus becomes an overall quality indicator for product, reflecting the effect of all significant variables according to each variable's density rate. In the latter case study, the suggested metric will be calculated and its performance will be evaluated. The next section explains a comparison-based approach for finding the machines that are the root cause of the detected anomalies.

\subsection{Peer-to-peer (P2P) comparison for anomaly identification}

For the fault diagnosis of manufacturing equipment involved in a multistage process, a peer set refers to a cluster of machines sharing the same throughput resources and environmental conditions. A Peer-to-Peer (P2P) comparison approach compares the performances of all peers within one peer set and evaluates the consistency of these peers, and classifies peers that show a large deviation to most the other peers as abnormal ones. In the current study, a peer set is defined as multiple machines in the same stage producing the same type of product that has been processed by the same machine in a previous stage. It is assumed that the products are being distributed between the machines randomly from one stage to the next. The peer-topeer comparison approach is applied to the product quality measurement data to identify the root causes of anomalies in a multistage manufacturing process. This method takes advantage of the fact that one upstream stage can diverge to multiple downstream processes. The root causes can be identified in a certain stage by evaluating performance consistency of a peer set sharing the same previous stage.

Figure 6 shows two possible cases in a two-stage manufacturing process in which one machine in stage 1 diverges to peer sets of multiple machines in stage 2 or vice versa, where multiple machines in stage 1 converge to one machine in stage 2 . The manufacturing routes from stage 1 to stage 2 are shown in blue arrows. In any of these two situations, evaluating the inconsistencies in the performance level of peers in one stage can lead to determining the root cause of quality issues. In peer set type 1 for example, if one or more than one of the machines performs significantly lower than the average of other peers, they will be the root cause of the anomaly. However, if all peers perform similarly, the existing anomaly can be associated with the machine in stage 1. Likewise, the peer comparison approach can be applied to the scenario of peer set type 2 in Figure 6, in which products manufactured by $\mathrm{M}_{1.1}, \mathrm{M}_{1.2}, \ldots, \mathrm{M}_{1 . n}$ are processed by the same second-stage equipment $\mathrm{M}_{2.1}$. The first sub-index indicates the stage number and the second sub-index indicates the machine number. This notation will be used throughout the paper. Later, a demonstration in this paper considers peer set type 1 , as it is the case for the dataset available for this study.

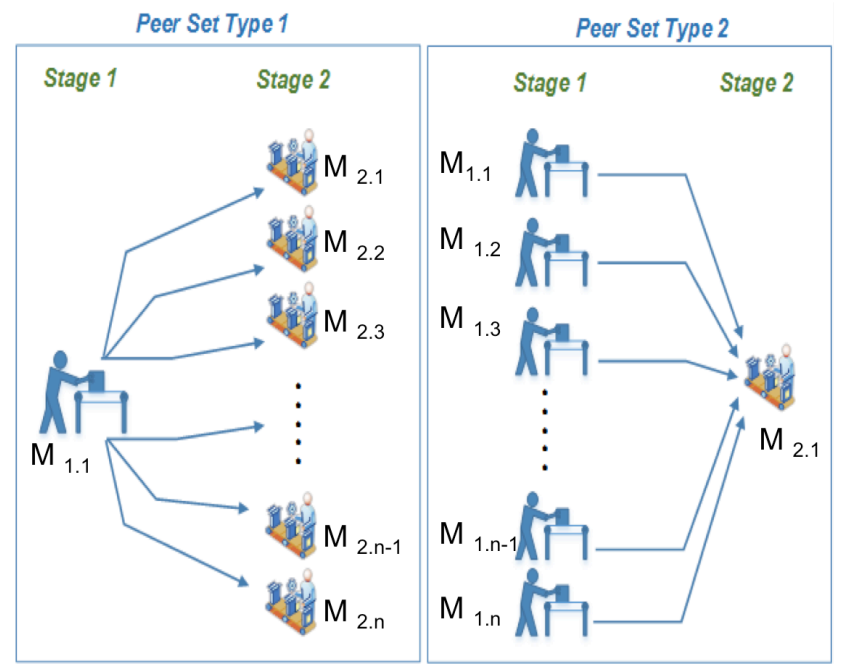

Figure 6. Peer sets in a two-stage manufacturing process for product type $\mathrm{A}$

For applying the peer comparison method to the available data set, two assumptions have been considered:

1. During a regular operation, more than half of stage 2 machines $\mathrm{M}_{2.1}, \mathrm{M}_{2.2} \ldots \mathrm{M}_{2 . \mathrm{n}}$ belonging to one peer set are in good condition.

2. Products manufactured by $\mathrm{M}_{1.1}$ are randomly distributed among machines at the second stage.

These assumptions are required to select the median value from all the manufacturing routes in one peer set and set it as the baseline value to be used for evaluating the machines in the peer set. The case being studied in this paper includes multiple two-stage manufacturing processes of peer set type 1 , and the evaluation is done for product type A.

Based on this type of manufacturing processes, all individual processes following the same first stage equipment at stage 2 are selected as in the same peer set. A given peer set includes $n(n>5)$ manufacturing routes all diverging from one machine in stage 1 , and ending in $n$ machines in stage 2 . At a specific time $t$, the product quality metrics of all the manufacturing routes are $\mathrm{q}_{1 \mathrm{t}}, \mathrm{q}_{2 \mathrm{t}}, \cdots, \mathrm{q}_{\mathrm{nt}}$. Then, $Q_{t}$ is calculated as the nominal value from $\mathrm{q}_{1 \mathrm{t}}, \mathrm{q}_{2 \mathrm{t}}, \cdots, \mathrm{q}_{\mathrm{nt}}$ following the rule of

$$
\mathrm{Q}_{\mathrm{t}}=\operatorname{median}\left(\mathrm{q}_{1 \mathrm{t}}, \mathrm{q}_{2 \mathrm{t}}, \ldots, \mathrm{q}_{\mathrm{nt}}\right)
$$

$Q_{t}$ is the nominal value representing the performance of the machine in stage $1\left(\mathrm{M}_{1.1}\right)$ for all the manufacturing routes in this peer set. Considering the scenario in which $\mathrm{M}_{1.1}$ is in good condition, it is expected that the median value of product quality metrics of all peers in stage 2 would be good based on the assumption that more than half of stage 2 machines belonging to one peer set are in good condition. Thus, it is reasonable to use $Q_{t}$ as the performance metric for stage 1 machine. When $Q_{t}$ is lower than the threshold of the allowable quality of products, an anomaly in $\mathrm{M}_{1.1}$ is 
detected. Subtracting $\mathrm{Q}_{\mathrm{t}}$ from $\mathrm{q}_{\mathrm{it}}$ gives a normalized metric for machines in stage 2 , as it indicates the difference between the performances of each machine with the median of all other machines in stage 2 . Let $\mathrm{R}_{\mathrm{it}}$ represent the performance metric for machine $i$ in stage 2 at time $t$. This metric can be defined as:

$$
\mathrm{R}_{\mathrm{it}}=\mathrm{q}_{\mathrm{it}}-\mathrm{Q}_{\mathrm{t}}
$$

If $\mathrm{R}_{\mathrm{it}}<0$, the quality of the products manufactured from route $\mathrm{i}$ (or by machine $\mathrm{i}$ in stage 2) at time $\mathrm{t}$ is lower than the nominal quality of product manufactured from all other routes. The smaller the value of $\mathrm{R}_{\mathrm{it}}$, the worse the condition of $M_{2 . i}$. When $R_{i t}$ is less than a certain threshold, an anomaly in $M_{2 . i}$ will be detected. The proper threshold should consider the product quality requirements and is not discussed in this paper. Instead, this paper is focused on the verification of the proposed method through observing the trend development of machine performance metrics and how it relates to possible maintenance actions in the manufacturing plant.

In the suggested approach, the stage 1 machine performance metric $Q_{t}$ is designed to represent the overall quality of the products manufactured by $\mathrm{M}_{1.1}$. Similarly, the stage 2 machine performance metric $\mathrm{R}_{\mathrm{it}}$ represents the deviation of $M_{2 . i}$ from the nominal performance of its peers.

In the next section of the paper, the results obtained by applying the suggested approach on the available data set is presented and explained.

\section{CASE STUDY: RESULTS AND DISCUSSIONS}

In this section, the results obtained by applying the suggested methodology to the available data are presented. The results include the identified anomaly root causes for different manufacturing routes with a relatively large number of samples.

The data under consideration is collected from a multistage manufacturing plant producing products with different specifications based on their intended applications. It contains the barcode for each product, product type, the machines' IDs that were used to produce each product in the last two stages of the process, 16 product quality measurements for each product, and the final quality of the products, whether it passed or failed. Table 1 shows the summary of the collected data set.

With the manufacturing process being multi-stage by nature, according to the maintenance experts at the specific industrial facility, the last two stages of the process are the most likely problematic stages and are therefore the focus of this case study. The products to be evaluated are of two categories: passed, rejected. Passed category includes those products that have passed quality check and are therefore within customer specifications. Those products are regarded as having good quality compared with products from the other category and are labeled as healthy class in this section. Rejected category includes those products that have failed quality check and are therefore out of customer specifications. Those products are regarded as having low quality comparing with products from the other category and are labeled as faulty class in this section.

\begin{tabular}{|l|l|}
\hline Product Information & Barcode, product type \\
\hline Manufacturing Path & $\begin{array}{l}\text { Machine ID in stages 1 and } \\
\text { Quality Measurements } \\
\text { each product }\end{array}$ \\
\hline $\begin{array}{l}\text { Product Quality Control } \\
\text { Result }\end{array}$ & $\begin{array}{l}\text { Flag determining whether } \\
\text { each product passed, } \\
\text { rejected or needed rework. }\end{array}$ \\
\hline $\begin{array}{l}\text { Duration of Data } \\
\text { Collection }\end{array}$ & 3 months \\
\hline Number of Samples & $210 \mathrm{~K}$ samples \\
\hline
\end{tabular}

Table 1. Summary of the collected dataset

To select critical variables, the variable correlation is studied for both the healthy and faulty class separately as it is unknown if the correlation relationship will change when product quality level differs. For example, if variable a and $\mathrm{b}$ are highly correlated for the healthy class but are not correlated for the faulty class, both variables need to be retained since they are both useful in abnormality detection and they contain no redundant information for one of the classes.

The correlation between each pair of variables is calculated for the product data taken from different quality groups separately. Pairs of variables having correlation coefficients larger than 0.9 are considered as highly correlated. For those highly correlated variables, only the variable with the higher Fisher value will be included in the later modeling stage.

Table 2 includes an example of the correlation coefficients of the highly correlated variable pair among the 16 product quality variables for the healthy class and the faulty class respectively.

Table 2 provides the linear correlation coefficient (Chun and Keleş 2010, 3-25) between each pair of the highly correlated variables (having a linear correlation coefficient larger than $0.9)$. Integers for V1 and V2 are the variable numbers in one correlated pair (variable numbers are labeled from 1 to 16 for the 16 quality variables) and Corr is the linear correlation coefficient between each variable pair V1 and V2. Tables of correlation values for both classes lead to the 


\begin{tabular}{|l|l|l|l|l|l|l|l|l|l|l|l|l|l|l|l|}
\hline Class & V1 & V2 & Corr & V1 & V2 & Corr & V1 & V2 & Corr & V1 & V2 & Corr & V1 & V2 & Corr \\
\hline Healthy & 1 & 14 & $\mathbf{0 . 9 1}$ & 2 & 16 & $\mathbf{0 . 9 3}$ & 3 & 15 & $\mathbf{0 . 9 5}$ & 6 & 7 & $\mathbf{0 . 9 6}$ & 8 & 9 & $\mathbf{0 . 9 7}$ \\
\hline Faulty & 1 & 14 & $\mathbf{0 . 9 3}$ & 2 & 16 & $\mathbf{0 . 9 6}$ & 3 & 15 & $\mathbf{0 . 9 4}$ & 6 & 7 & $\mathbf{0 . 9 4}$ & 8 & 9 & $\mathbf{0 . 9 6}$ \\
\hline
\end{tabular}

Table 2. Correlation values of highly correlated inspection variables

\begin{tabular}{|l|l|l|l|l|l|l|l|l|l|l|l|l|l|l|l|}
\hline V1 & V2 & V3 & V4 & V5 & V6 & V7 & V8 & V9 & V10 & V11 & V12 & V13 & V14 & V15 & V16 \\
\hline 0.46 & 0.26 & 0.10 & 0.01 & 0 & 0 & 0 & 0.01 & 0.01 & 0 & 0.39 & 0.22 & 0.11 & 0.50 & 0.10 & 0.28 \\
\hline
\end{tabular}

Table 3. Fisher values of product type A inspection variables

conclusion that high correlations of variable pairs are consistent for both classes. Therefore, it is reasonable to reduce the dimension by removing one variable from each highly correlated variable pair.

Table 3 gives the Fisher value of each inspection variable using Fisher criterion and those highly correlated ones are highlighted in the same color. For highly correlated variables, only one variable in a correlated pair is preserved for later analysis. Based on the Fisher selection, those variables having a higher Fisher value than the mean Fisher value of all variables are selected. For the product type A being studied here, the final selected critical variables V11, V12, V13, V14 and V16, are indicated in red.

Using the selected critical variables, peer comparison performance metrics of machines in stages 1 and 2 for product type A are shown in Figure 7 and Figure 8. Results for potentially problematic machines are included and those of high performance machines are selectively presented due to their similar trends. Using the performance metrics of each stage, the presented approach enabled the fault diagnosis of a two-stage manufacturing process by relying only on the product quality measurements. As the first step, the product quality metrics $\mathrm{q}_{1 \mathrm{t}}, \mathrm{q}_{2 \mathrm{t}}, \ldots, \mathrm{q}_{24 \mathrm{t}}$ for the 24 manufacturing routes (from $\mathrm{M}_{1.1}$ to $\mathrm{M}_{2.1}, \mathrm{M}_{2.2} \ldots, \mathrm{M}_{2.24}$ ) were calculated using Eq. (2). Then, $Q_{t}$ was calculated as the median of $q_{1 t}, q_{2 t}, \ldots, q_{24 t}$ to represent the performance metric of $\mathrm{M}_{1.1}$. The metrics for the 24 machines in stage 2 $\left(\mathrm{M}_{2.1}, \mathrm{M}_{2.2} \ldots, \mathrm{M}_{2.24}\right)$ were obtained using Eq. (4). These metrics, namely $R_{1 t}, R_{2 t}, \ldots$ and $R_{24 t}$, are selectively shown in Figure 8.

It is observed from Figure 8 that the machine performance metrics of $\mathrm{M}_{2.1}$ and $\mathrm{M}_{2.2}$ decreased around August 18th, 2012. At this time, the second-stage equipment $M_{2.1}$ and $\mathrm{M}_{2.2}$ were identified as the root cause of the anomalies in the production due to the developing trends of their performance metrics. This conclusion agrees with the fact that $\mathrm{M}_{2.1}$ and $\mathrm{M}_{2.2}$ were no longer used to manufacture this product type starting from the end of August.

A more important observation from Figure 7 is that starting from mid-October, the performance metric of the first-stage

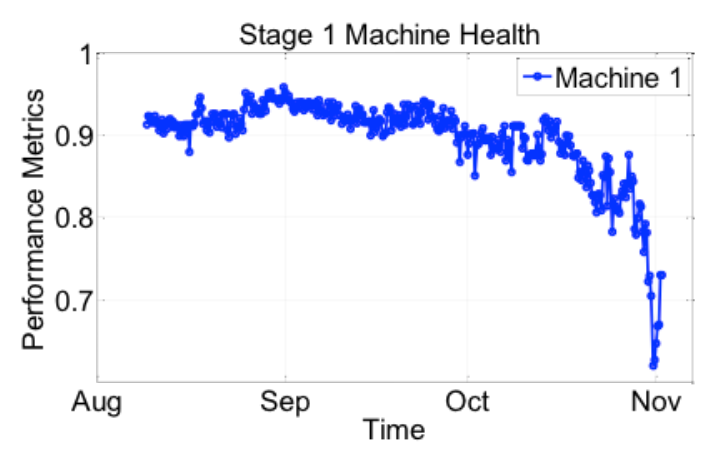

Figure 7. Peer comparison results of stage 1 for product type A
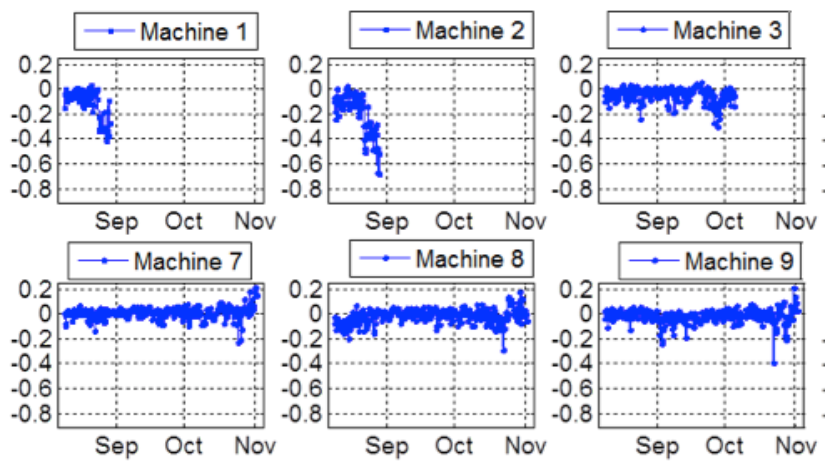

Figure 8. Selective peer comparison results of stage 2 for product type A

equipment $\mathrm{M}_{1.1}$ began a gradual decrease. The performance metric was originally at a high level around 0.9 and later began to descend to around 0.8 . Then the decreasing trend became more severe until it dropped below 0.65. This agrees with one's expectation that the equipment anomaly usually develops over time. The anomaly at its beginning stage results in a gradual decrease in the health value or health metric. Since the anomaly in this stage is still incipient, the quality of the manufactured products may remain within the specifications. The magnitude of the 
negative slope value then starts to increase, as the anomaly gets more severe. Such behavior can be clearly seen in the health value of $\mathrm{M}_{1.1}$ in Figure 7 . With the dataset provided, around the beginning of November, this machine was no longer dedicated to manufacturing any product, which can be a plausible indication that maintenance actions were performed on this machine.

Monitoring the health value of these machines can help the maintenance crew to detect the anomalies when they are still incipient. This leads to more proactive maintenance actions and the avoidance of failures, which can significantly reduce the costs of both equipment repair and production downtime. It is notable that the presented method does not require additional sensors and equipment to collect inprocess measurements; it only relies on the production quality data.

\section{CONCLUSIONS}

This paper introduces a novel methodology for identifying the root cause of the anomalies that affect the product quality in multi-stage manufacturing processes. The key advantage of the proposed method over previously developed methods is that the proposed technique only relies on the product quality measurement from the final stage, given basic manufacturing records and the presence of peer sets, instead of requiring availability of measurement data at each manufacturing stage. Implementation is also simple and computationally efficient, making it effective in helping the manufacturing and maintenance personnel with improved productivity. The methodology was validated and demonstrated using an industrial case study. The obtained results are proven to be effective in identifying the root cause of process anomalies and showed consistency with the real-world behavior of the machines involved in the manufacturing process.

\section{FUTURE WORK}

The methodology has the potential to be effective in various types of manufacturing processes. Thus, there are several directions that future work can be taken to further enhance its value. One area of future research is to design a predictive manufacturing scheme based on the limited data resources in product quality measurement. Such a scheme can be further developed based on a peer-to-peer comparison approach to incorporate the intelligent maintenance systems into the manufacturing processes. While two key manufacturing stages have been considered in this study, measurement from other manufacturing stages becomes necessary when utilizing this methodology to study more than 2 manufacturing stages. Future work on designing different sensor distribution and process measurement strategies will facilitate diagnosis in multistage manufacturing process with less data resource and associated implementation cost.

\section{REFERENCES}

Asadzadeh, S., \& Aghaie, A. (2008). A Review of Cause Selecting Control Charts in Multistage Processes. Proceedings of the 38th International Conference on Computers and Industrial Engineering, Vols 1-3, 16571665.

Asadzadeh, S., \& Aghaie, A. (2012). Improving the Product Reliability in Multistage Manufacturing and Service Operations. Quality and Reliability Engineering International, 28(4), 397-407. doi: 10.1002/qre.1254

Ceglarek, D., Shi, J., \& Wu, S. M. (1994). A KnowledgeBased Diagnostic-Approach for the Launch of the Auto-Body Assembly Process. Journal of Engineering for Industry-Transactions of the Asme, 116(4), 491499. doi: Doi 10.1115/1.2902133

Chan, W. M., \& Ibrahim, R. N. (2004). Evaluating the quality level of a product with multiple quality characterisitcs. International Journal of Advanced Manufacturing Technology, 24(9-10), 738-742. doi: 10.1007/s00170-003-1751-6

Chan, W. M., Ibrahim, R. N., \& Lochert, P. B. (2005a). Evaluating the product quality level under multiple Ltype quality characteristics. International Journal of Advanced Manufacturing Technology, 27(1-2), 90-95. doi: 10.1007/s00170-004-2158-8

Chan, W. M., Ibrahim, R. N., \& Lochert, P. B. (2005b). Quality evaluation model using loss function for multiple S-type quality characteristics. International Journal of Advanced Manufacturing Technology, 26(12), 98-101. doi: 10.1007/s00170-003-1980-8

Hawkins, D. M. (1993). Regression Adjustment for Variables in Multivariate Quality-Control. Journal of Quality Technology, 25(3), 170-182.

$\mathrm{Hu}$, S. J., \& Wu, S. M. (1992). Identifying Sources of Variation in Automobile Body Assembly Using Principal Component Analysis. Transactions of the North American Manufacturing Research Institution of Sme, Vol 20, 1992, 311-316.

Jin, J., \& Shi, J. (1999). State space modeling of sheet metal assembly for dimensional control. Journal of Manufacturing Science and Engineering-Transactions of the Asme, 121(4), 756-762. doi: Doi 10.1115/1.2833137

Kapur, K. C., \& Cho, B. R. (1996). Economic design of the specification region for multiple quality characteristics. Iie Transactions, 28(3), 237-248. doi: Doi 10.1080/07408179608966270

Liu, S. C., \& Hu, S. J. (1997). Variation simulation for deformable sheet metal assemblies using finite element methods. Journal of Manufacturing Science and Engineering-Transactions of the Asme, 119(3), 368374. doi: Doi 10.1115/1.2831115

Lowry, C. A., Woodall, W. H., Champ, C. W., \& Rigdon, S. E. (1992). A Multivariate Exponentially Weighted 
Moving Average Control Chart. Technometrics, 34(1), 46-53. doi: Doi 10.2307/1269551

Lucas, J. M., \& Saccucci, M. S. (1990). Exponentially Weighted Moving Average Control Schemes Properties and Enhancements. Technometrics, 32(1), 112. doi: Doi 10.2307/1269835

Macgregor, J. F., \& Kourti, T. (1995). Statistical ProcessControl of Multivariate Processes. Control Engineering Practice, 3(3), 403-414. doi: Doi 10.1016/09670661(95)00014-L

Neubauer, A. S. (1997). The EWMA control chart: Properties and comparison with other quality-control procedures by computer simulation. Clinical Chemistry, 43(4), 594-601.

Page, E. S. (1954). Continuous Inspection Schemes. Biometrika, 41(1-2), 100-\&. doi: DOI 10.1093/biomet/41.1-2.100

Rato, T. J., \& Reis, M. S. (2011). Statistical Process Control of Multivariate Systems with Autocorrelation. 21st European Symposium on Computer Aided Process Engineering, 29, 497-501.

Reynolds, M. R., Amin, R. W., \& Arnold, J. C. (1990). Cusum Charts with Variable Sampling Intervals Response. Technometrics, 32(4), 393-396.

Reynolds, M. R., Amin, R. W., Arnold, J. C., \& Nachlas, J. A. (1988). Xbar Charts with Variable Sampling Intervals. Technometrics, 30(2), 181-192. doi: Doi $10.2307 / 1270164$

Scouse, R. A. (1985). Introduction to Statistical QualityControl. Plastics \& Rubber International, 10(1), 30-32.
Shu, L. J., \& Tsung, F. (2000). Multistage process monitoring and diagnosis. Proceedings of the 2000 Ieee International Conference on Management of Innovation and Technology, Vols 1 and 2, 881-886.

Tsung, F. G., Li, Y. T., \& Jin, M. (2006). Statistical process control for multistage manufacturing and service operations: A review. 2006 Ieee International Conference on Service Operations and Logistics, and Informatics (Soli 2006), Proceedings, 752-757. doi: Doi 10.1109/Soli.2006.329084

Wolbrecht, E., D'Ambrosio, B., Paasch, R., \& Kirby, D. (2000). Monitoring and diagnosis of a multistage manufacturing process using Bayesian networks. Ai Edam-Artificial Intelligence for Engineering Design Analysis and Manufacturing, 14(1), 53-67.

Woodall, W. H., \& Ncube, M. M. (1985). Multivariate Cusum Quality-Control Procedures. Technometrics, 27(3), 285-292. doi: Doi 10.2307/1269710

Zhou, S. Y., Ding, Y., Chen, Y., \& Shi, J. J. (2003). Diagnosability study of multistage manufacturing processes based on linear mixed-effects models. Technometrics, 45(4), 312-325. doi: 10.1198/004017003000000131

Zhou, S. Y., Huang, Q., \& Shi, J. J. (2003). State space modeling of dimensional variation propagation in multistage machining process using differential motion vectors. Ieee Transactions on Robotics and Automation, 19(2), 296-309. doi: 10.1109/Tra.2003.808852 